\title{
Protocolo: análisis de los estudios universitarios oficiales en España
}

\section{Protocol: analysis of official university studies in Spain}
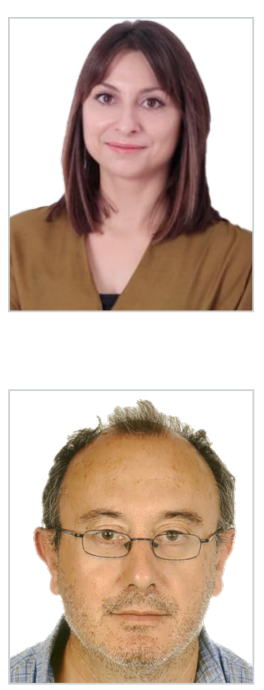

Leticia Rodríguez Fernández. Doctora en Ciencias de la Información (UCM, 2012), Licenciada en Publicidad y Relaciones Públicas (UVA, 2007), Máster en Comunicación de Instituciones Públicas y Políticas (UCM, 2008), Máster Universitario en Protocolo (UNED, 2019). Docente en la Universidad Antonio Nebrija en el campo de la comunicación organizacional. Directora del Grado en Grado en Comunicación Corporativa, Protocolo y Organización de Eventos (2017-actualidad). Experiencia profesional de 7 años como consultora de comunicación. Sus líneas de investigación se centran en la comunicación organizacional, con especialización en el área digital y el estudio de la propaganda y la desinformación.

Universidad Antonio Nebrija, España

Irodrigu@nebrija.es

ORCID: 0000-0002-7472-5472

Antonio Castillo Esparcia. Catedrático de Universidad. Licenciado y doctor en Comunicación por la Universitat Autónoma de Barcelona (UAB). Autor de más de 170 publicaciones entre artículos en revistas, libros y capítulos de libros en editoriales nacionales y extranjeras. Director del Proyecto de Investigación Lobby y Comunicación, financiado por el Programa Estatal de I+D+i (CSO2016-79357-R). Director del Grupo de Investigación Las Relaciones Públicas en la pequeña y Mediana Empresa. Presidente de la Asociación de Investigadores en Relaciones Públicas (AIRP). En la actualidad, es el director del Departamento de Comunicación Audiovisual y Publicidad y Coordinador del Máster Dirección Estratégica e Innovación en Comunicación. Asimismo, es evaluador de la Agencia Nacional de Evaluación de la Calidad y Acreditación (ANECA), Agència per a la Qualitat del Sistema Universitari de Catalunya (AQU), Agencia de Calidad del Sistema Universitario Vasco (UNIBASQ) y Agencia Estatal de Investigación.

Universidad de Málaga, España

acastilloe@uma.es

ORCID: 0000-0002-9751-8628

Recibido: 31/12/2019 - Aceptado: 17/04/2020

\section{Resumen:}

Los estudios oficiales en el ámbito del protocolo son relativamente nuevos, si atendemos a otras disciplinas, pero gozan de solidez y gran proyección, dada la necesidad que tienen las organizaciones de contar con un perfil de estas características. No obstante, el nú-
Received: 31/12/2019 - Accepted: 17/04/2020

\section{Abstract:}

Official studies in the scope of protocol are relatively new compared to other disciplines; however, they are consistent and relevant, given the need for organisations to have such a profile. Nevertheless, the number of official studies is still limited, making it an area of inter-

Cómo citar este artículo:

Rodríguez Fernández, L.; Castillo Esparcia, A. (2020). Protocolo: análisis de los estudios universitarios oficiales en España. Doxa Comunicación, 30, pp. 211-227. 
mero de estudios oficiales resulta aún limitado, lo que la convierte en un área de interés por su potencial crecimiento. Se propone en este trabajo un análisis de las titulaciones oficiales de grado y máster para conocer sus características, contenidos, competencias y perfiles profesionales. Se observa entre las conclusiones, poca diferencia entre la oferta formativa de grado y máster y un enfoque mayor en el área de eventos que en el propio protocolo.

\section{Palabras clave:}

Protocolo; comunicación; grado; máster; universidad. est due to its potential growth. This paper proposes an analysis of the official titles of the bachelor's and master's degrees to know their characteristics, contents, competencies, and professional profiles. Among the conclusions, there is little difference between the offer of undergraduate and master's training, and there is a greater focus on the area of events rather than on protocol itself.

Keywords:

Protocol; communication; degree; master's degree; university.

\section{Estado de la cuestión}

\subsection{Protocolo y eventos en las titulaciones en comunicación}

El ámbito de la comunicación en las organizaciones aborda todo el complejo marco de la dirección y gestión de la planificación y las herramientas de comunicación. Diversos autores han insertado el protocolo como herramienta de relaciones públicas puesto que de su ejercicio se potencia, a través de los eventos, una de las modalidades más efectivas de la comunicación como es la comunicación interpersonal (Otero, 2009; Marín Calahorro, 2000, Castillo-Esparcia y Fernández-Torres; 2015).

Tradicionalmente, el responsable de protocolo era un profesional formado a través de su propia experiencia y de la de sus compañeros de profesión. En algunos casos, estos expertos procedían de licenciaturas como Publicidad y Relaciones Públicas, Turismo o Derecho. En otros, la profesión se ejercía sin ningún estudio oficial previo, y se obtenía el conocimiento formativo a través de títulos propios, cursos, congresos y la reducida pero valiosa literatura científica de los próceres académicos.

Desde el ámbito de la comunicación, el protocolo formaba parte de los contenidos de algunas asignaturas del Grado en Publicidad y Relaciones Públicas. Así se observa en el Libro Blanco. Título de Grado en Comunicación, publicado en 2003 con el objetivo de establecer un documento marco para la adaptación de los estudios españoles en su adaptación hacia el Plan Bolonia. En el título de Publicidad y Relaciones Públicas se recoge dentro de la materia "Fundamentos, estructuras y organización de la comunicación publicitaria y de las relaciones públicas la "capacidad para la organización, ejecución y evaluación de eventos, cuidando especialmente su diseño, control de costes y protocolo".

En 2005, Sierra y Sotelo (2008) identificaron que 11 de las 41 universidades con estudios de Ciencias de la Información ofertaban asignaturas troncales, optativas, básicas y de libre elección sobre protocolo y 10 de los centros analizados ofrecían estudios de protocolo en postgrados.

Hernández, Losada y Matilla (2009) ahondaron en la oferta de posgrado de comunicación corporativa en España. Se concluye que solo dos títulos incluían en su denominación las acepciones "relaciones públicas" y "comunicación corpo- 
rativa", decantándose por otros términos como "protocolo" u "organización de eventos". Los eventos, además, figuraban en el título de la tercera parte de los estudios analizados.

Herrero (2013) realiza en su Trabajo Fin de Máster un análisis más amplio que recoge los grados, postgrado y títulos de experto universitario en protocolo. De las 81 universidades examinadas, 47 contaban con estudios en comunicación y 24 ofrecían en dichos estudios asignaturas de organización de eventos, protocolo y/o relaciones institucionales. Señala la autora que entre 2005 y 2013 el protocolo, unidos a la organización de eventos y a las relaciones institucionales, alcanza mayor notoriedad dentro de las universidades que pasan de ofertar 8 posgrados a 13. (Herrero; 2013: 25).

A nivel internacional, Herrero y Perelló (2016) comparan los planes de estudios de 31 grados del área del protocolo y la organización de eventos en 9 países de la Unión Europea (Alemania, Austria, Dinamarca, España, Francia, Irlanda, Islandia, Portugal y Reino Unido). Entre las conclusiones más interesantes destaca que las asignaturas "Técnicas de Organización de Eventos", "Planificación de Eventos" e "Industria de Eventos" figuran en casi todos los planes de estudios. No obstante, los autores indican que las "materias más específicas de algún ámbito de la organización de eventos se ven reflejadas en menor medida" (p.452). Esto sugiere que se elude de alguna manera la especialización, inclinándonos hacia asignaturas más genéricas y amplias en contenido. Asimismo, los idiomas y las nuevas tecnologías no se ofertan en la mayoría de titulaciones.

Portugal, Becerra y Victoria (2016) realizan un análisis similar en las titulaciones implantadas en España. Se observa que parte de sus planes de estudio son impartidos en grados de las mismas universidades por lo que podría extraerse que hay cierta necesidad de aprovechar al profesorado en su desarrollo. Los autores además ahondaron en la bibliografía de las asignaturas, profundizando sobre las guías docentes, para concluir que los contenidos se asentaban sobre publicaciones modernas, dejando de lado a los padres del protocolo.

Saavedra y Perlado (2007) analizan, desde el enfoque de la dirección de comunicación, los estudios de comunicación en España, incluyendo grados, posgrados y títulos propios. El protocolo y el ceremonial figuran en gran parte de los estudios como competencia específica y otorgan la segunda posición de peso en contenido a la "esfera de marca corporativa" en la que se incluye la dirección, estructura y desarrollo de eventos, protocolo, ceremonial y simbología.

\subsection{Perspectiva de los profesionales de protocolo}

De manera usual, la organización de eventos y el protocolo han sido asumidos por profesionales procedentes de estudios de información que complementaban esta formación con postgrados o expertos en protocolo. Según el "Informe Anual de la Profesión Periodística 2017", entre las principales actividades de los autónomos en comunicación, la organización de eventos representa el 23.9\%. También en el "Estado de la comunicación en España” (2018) encontramos actividades relacionadas directa o indirectamente con el protocolo como los eventos, que supone el $2.3 \%$ de las tareas de los profesionales de la comunicación, las relaciones con el gobierno, asuntos públicos y lobbying (18,6\%) o la comunicación internacional $(7,5 \%)$.

Según el informe "El Sector Eventos en España” elaborado por Event Managers Association-EMA- (2018), el perfil actual del event manager en España es mayoritariamente femenino (76\%), senior (entre 40 y 49 años) y con un elevado nivel de 
estudios. En relación a los estudios universitarios cursados, el 31,7\% de los perfiles procedía del Grado en Turismo y un 29,6\% del Grado en Publicidad y Relaciones Públicas.

España es además una de las grandes potencias en organización de eventos. Según The International Association Meetings Market 2017, es el cuarto en el mundo con mayor número de eventos y el segundo en estimación de participación. Este buen posicionamiento se recoge también en otros informes sectoriales como "El evento como generador de valor estratégico" (2018) elaborado por Grupo Eventoplus, que refleja que las empresas prevén un crecimiento del 3,8\% en el presupuesto dedicado al sector, que se traducirá en un aumento del 10,1\% en las plantillas.

En este sentido, el profesional del protocolo debe disponer de profundos conocimientos sobre el papel de la comunicación en las organizaciones como herramienta de proyección de la idiosincrasia de la entidad.

\section{Objetivos y metodología}

El presente trabajo busca analizar los estudios oficiales de grado y máster en protocolo en España con el objetivo de conocer sus características básicas. Se pretende identificar:

O1: Aspectos formales de estos estudios: denominación, número de créditos, idioma y modalidades de impartición.

O2: El carácter de las universidades (públicas y/o privadas) que ofrecen estas titulaciones

O3: Los contenidos prevalentes en los planes de estudios

O4: Las competencias específicas en el ámbito del protocolo

O5: Los perfiles profesionales derivados

Se ha realizado, en primer lugar, una investigación exploratoria sobre trabajos académicos y artículos en revistas indexadas del área del protocolo y la comunicación corporativa e institucional. Dada la estrecha relación de este trabajo con el campo profesional, también se han recogido otras fuentes de valor como informes y estudios profesionales o entrevistas a representantes sectoriales. Esta revisión bibliográfica ha permitido contextualizar la evolución de los estudios universitarios en protocolo en España y realizar un estudio de antecedentes de los trabajos publicados.

A continuación y con la intención de realizar un análisis de contenido de las titulaciones universitarias oficiales en protocolo se han extraído del Registro de Universidades, Centros y Títulos (RUCT) todos los títulos de grado y máster universitarios del área. Esta consulta se realizó en el mes de marzo de 2019 y de ella se obtuvieron datos básicos para la ficha de análisis como el año de verificación, el año de implantación, total de créditos, nombre de la universidad y centro en el que se imparte y competencias específicas. A lo largo de todo el trabajo se ha seguido la misma ordenación de grados y máster utilizada en el RUCT.

Cabe destacar que se han tomado como referencia únicamente los estudios oficiales, excluyendo de la muestra de análisis los títulos propios, los expertos y otras titulaciones similares ofertados por universidades y escuelas de negocio. Esta delimitación atiende a la propia lógica universitaria: los estudios oficiales pasan por un proceso de verificación, seguimiento 
y acreditación que garantizan su calidad. Por tanto, tienen un valor académico mayor y requieren de un proceso de planificación más elaborado que un título propio, que depende únicamente de la institución creadora.

Finalmente y sobre esta información se realizó un análisis de contenido en base a los objetivos planteados para esta investigación, que se recogieron en la siguiente ficha:

Tabla 1. Metodología. Ficha de análisis

\begin{tabular}{|c|c|}
\hline \multicolumn{2}{|c|}{ DATOS BASICOS- GRADO/MÁSTER } \\
\hline NOMBRE DEL TÍTULO & \\
\hline \multicolumn{2}{|l|}{ UNIVERSIDAD } \\
\hline \multicolumn{2}{|l|}{ FACULTAD } \\
\hline \multicolumn{2}{|l|}{ CARÁCTER UNIVERSIDAD } \\
\hline \multicolumn{2}{|c|}{ CIUDAD EN LA QUE SE IMPARTE } \\
\hline \multicolumn{2}{|l|}{ AÑO DE VERIFICACIÓN } \\
\hline \multicolumn{2}{|l|}{ AÑO DE IMPLANTACIÓN } \\
\hline \multicolumn{2}{|l|}{ MODALIDAD } \\
\hline \multicolumn{2}{|l|}{ IDIOMAS } \\
\hline \multicolumn{2}{|l|}{ DATOS ACADÉMICOS } \\
\hline \multicolumn{2}{|l|}{ TOTAL CRÉDITOS } \\
\hline \multicolumn{2}{|l|}{ TOTAL DE ASIGNATURAS } \\
\hline \multicolumn{2}{|c|}{ TOTAL DE ASIGNATURAS SOBRE PROTOCOLO } \\
\hline \multicolumn{2}{|c|}{ TOTAL CRÉDITOS EN EL ÁREA DE PROTOCOLO } \\
\hline LISTADO DE ASIGNATURAS & Total créditos de cada asignatura \\
\hline \multicolumn{2}{|c|}{ LISTADO DE COMPETENCIAS ESPECÍFICAS EN LAS QUE SE MENCIONA DIRECTAMENTE EL PROTOCOLO } \\
\hline \multicolumn{2}{|l|}{ SALIDAS PROFESIONALES } \\
\hline \multicolumn{2}{|l|}{ COMENTARIOS } \\
\hline
\end{tabular}

Fuente: elaboración propia 
Para ahondar en la información académica (plan de estudios) nos remitimos a la página web de cada titulación. Una vez extraídas todas las asignaturas con contenido en protocolo se procedió a su categorización atendiendo a la especialización: historia del ceremonial/protocolo, protocolo institucional, protocolo empresarial y social, protocolo internacional y diplomacia, derecho premial y nobiliario, organización de eventos y protocolo y otros.

Con esta clasificación se buscaba conocer cuáles son los contenidos más abordados en los estudios oficiales. Cabe destacar que fue necesario, en algunos casos, consultar las guías docentes ya que sus descripciones no resultaban suficientemente claras.

Para conocer hacia dónde se orientan los esfuerzos de los estudios oficiales, se han categorización las competencias específicas tomando como referencia tres objetivos docentes básicos:

1. Vinculación del protocolo y el mundo profesional/social/empresarial: competencias que ofrecen capacidades específicas sobre dinámicas profesionales o sociales propias del sector. Conocimientos relacionados con el funcionamiento de un departamento de protocolo, medios de comunicación u otros organismos, así como la responsabilidad social y su aplicación en los eventos.

2. Aplicación práctica del protocolo/ceremonial/simbología: aplicación del protocolo en sus distintas especialidades.

3. Conocimientos teóricos del protocolo: conocimientos y comprensión de las bases teóricas del protocolo.

Finalmente se han tratado de extraer las salidas profesionales más citadas tanto en protocolo como en comunicación y en eventos.

Dado el valor que también tienen los títulos propios, se ha recogido una relación con la oferta formativa actual. Estos no formarán parte de la muestra de análisis pero se tendrán en cuenta para establecer una posible comparativa sobre aspectos menores con los títulos oficiales. En este caso, se han realizado varias búsquedas en Google con las keywords "Experto en protocolo", "Máster en protocolo", “Título propio en protocolo", limitando las búsquedas a las 3 primeras páginas de Google. Se ha delimitado la selección únicamente a aquellos títulos impartidos por universidades, centros adscritos y escuelas de negocio.

De ellas se ha obtenido información básica como nombre de la titulación, universidad, número de créditos, ciudad en la que se imparte, modalidad y url de los estudios. Se pretende tener una estimación del número de títulos propios, especialistas y diplomas de especialización no oficiales que se ofertan en la actualidad.

\section{Resultados: análisis de los estudios oficiales}

\section{1. resultados de los estudios de grado}

El Registro de Universidades, Centros y Títulos (RUCT) recoge en total 14 titulaciones, de las cuales siete son grados universitarios. Seis de estos grados están implantados en la actualidad, no obstante durante la realización del trabajo se observa que la Universidad de les Illes Balears indica que su titulación está en extinción. Aún así se ha recogido este grado en 
el análisis ya que está siendo impartido en estos momentos. No sucede lo mismo con el grado de la Universidad Europea que solicitó el curso pasado su extinción y ya no figura en su página web.

Tabla 2. Resumen de los grados oficiales que se analizarán en el trabajo

\begin{tabular}{|l|l|}
\hline \multicolumn{1}{|c|}{ GRADOS } & \multicolumn{1}{c|}{ UNIVERSIDAD } \\
\hline Grado en Comunicación Corporativa, Protocolo y Organización de Eventos & Universidad Antonio Nebrija \\
\hline Grado en Organización de Eventos, Protocolo y Relaciones Institucionales & Universidad Miguel Hernández de Elche \\
\hline Grado en Protocolo, Organización de Eventos y Comunicación Corporativa & Universidad Rey Juan Carlos \\
\hline Grado en Protocolo y Organización de Eventos & Universidad Camilo José Cela \\
\hline Grado en Protocolo y Organización de Eventos & Universidad del Atlántico Medio \\
\hline Grado en Protocolo y Organización de Eventos & Universitat de les Illes Balears \\
\hline
\end{tabular}

Fuente: Elaboración propia. Información extraída del RUCT

Todos los grados presentan el término "protocolo" en su denominación. No obstante siempre se relaciona con otras áreas de conocimiento como la comunicación, las relaciones institucionales y la organización de eventos, el campo más relevante ya que figura en la denominación de las seis titulaciones.

La posición del término en la denominación, que marca el orden de importancia, varía según la universidad. Cinco de ellos, es decir, el 91\% posicionan el protocolo como primer campo de conocimiento, frente a la Universidad Antonio Nebrija que posiciona la comunicación corporativa. Esta, junto con la Universidad Rey Juan Carlos son las únicas que añaden la comunicación corporativa como área vinculada. Igualmente, la Universidad Miguel Hernández incorpora las relaciones institucionales.

La primera universidad en solicitar la verificación de su titulación fue la Universidad Camilo José Cela que consiguió la misma en 2010. Le seguirán la Universidad Miguel Hernández (2012), la Universidad Europea (2013), Universidad Rey Juan Carlos (2015), Universidad Illes Balears (2015), Universidad Antonio Nebrija (2017) y Universidad del Atlántico (2017).

En relación al carácter de las instituciones, solo dos de ellas son públicas, frente a tres universidades privadas y un centro adscrito. La adscripción de los grados a las facultades es bastante dispersa: dos de las titulaciones se recogen en facultades de comunicación, dos en facultades de ciencias sociales, una en un instituto especializado en estudios de protocolo y otro en una escuela universitaria de turismo.

Madrid es la ciudad con mayor oferta de grados en protocolo (50\%) y a ella se añaden Las Palmas de Gran Canaria, Alicante y Palma de Mallorca. Todos los grados presentan la modalidad presencial y la Universidad Miguel Hernández de Elche, la Universidad Camilo José Cela y la Universidad del Atlántico Medio ofrecen también la modalidad a distancia. Todos los títulos se oferta en castellano y la Universidad Rey Juan Carlos es la única que permite la posibilidad de cursar la titulación en inglés.

Los grados presentan 240 créditos repartidos en 4 años, a excepción de la Universidad del Atlántico Medio cuyo título tiene 180 ECTS a cursar en 3 años. La Universidad Rey Juan Carlos ofrece la posibilidad de simultanear estudios con el Grado 
en Relaciones Internacionales, al igual que la Universidad Nebrija que añade además el Grado en Publicidad y Relaciones Públicas y el Grado en Periodismo.

Encontramos el mayor porcentaje de contenidos de protocolo en el plan de estudios de la Universidad Miguel Hernández de Elche (30\%). El mismo porcentaje se puede alcanzar en la Universitat de Illes Balears si es escoge en el itinerario de optativas únicamente asignaturas de protocolo. La Universidad Rey Juan Carlos es la que presenta menor contenido de protocolo en su plan de estudios (17,5\%).

Como se avanzó en la metodología, se han categorizado las asignaturas en base a su especialización diferenciando historia del ceremonial/protocolo, protocolo institucional, protocolo empresarial y social, protocolo internacional y diplomacia, derecho premial y nobiliario, organización de eventos y protocolo y otros. En esta clasificación se han tenido en cuenta las 65 asignaturas que contienen protocolo ofertadas por las 6 instituciones:

- La categorización con mayor número de asignaturas es el área de organización de eventos y protocolo. El 35,3\% de las asignaturas analizadas corresponden a este campo.

- Historia del ceremonial/protocolo figura en todos los planes de estudio, generalmente con una carga de 6 ECTS $(9,2 \%)$. Solo dos universidad rebajan la dedicación: la Universidad Miguel Hernández le otorga 4.5 ECTS. y la Universidad del Atlántico Medio 3 ECTS.

- El 12.3\% de las asignaturas ofertadas están relacionadas con el protocolo institucional, acepción más generalizada aunque también se recoge "protocolo oficial" o "ceremonial del Estado".

- El 10.7\% de las asignaturas abordan el derecho premial, nobiliario, la héraldica y/o la vexilología

- El 7.6\% de las asignaturas están relacionadas con el protocolo empresarial y social. Se han excluido otras que seguramente incorporarán el protocolo pero que en sus acepciones enfatizan los eventos como "Eventos sociales" o “Eventos empresariales y de marketing" por lo que tienen mayor vinculación con este campo.

- Igualmente el $7.6 \%$ de las asignaturas son del área de protocolo internacional o diplomacia.

- Finalmente, y por dispersión, se ha incluido una última categorización "otros" que recoge 11 asignaturas y supone el 16,9\% de las materias analizadas. En ella se recopilan las asignaturas de protocolo especializadas en algún ámbito como el académico, el religioso, el militar o el deportivo así como legislación o idiomas. Este último campo es el más novedoso ya que es ofertado únicamente por dos universidades: la Universidad del Atlántico Medio que ofrece "Inglés para profesionales del Protocolo y la Organización de Eventos" y la Universitat de les Illes Balears que ofrece "Catalán para protocolo y eventos". 
Ilustración 1. Gráfico: contenidos más ofertados en grados oficiales en protocolo

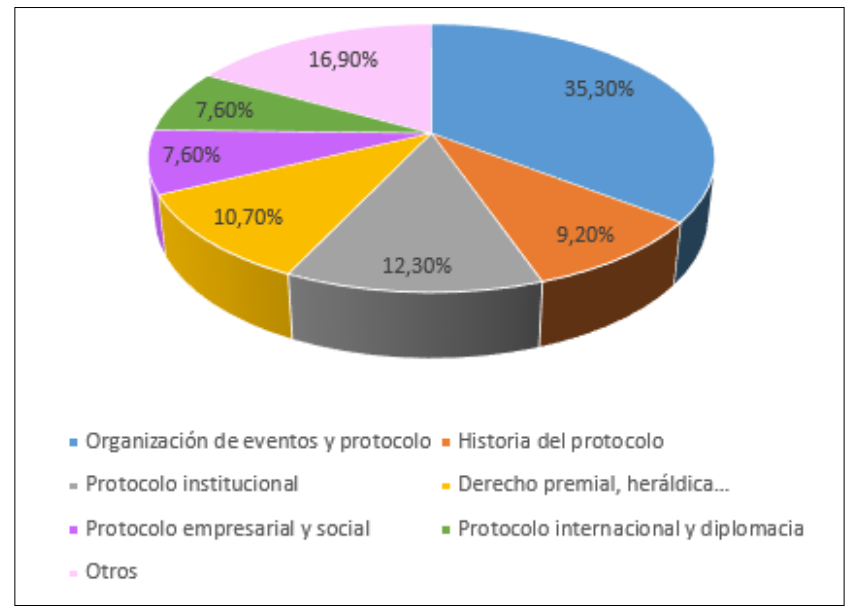

Fuente: elaboración propia

Las competencias específicas de protocolo varían considerablemente entre las universidades:

Tabla 3. Competencias específicas en cada grado oficial en protocolo

\begin{tabular}{|l|l|c|}
\hline \multicolumn{1}{|c|}{ Universidad } & \multicolumn{1}{c|}{ Grado } & $\begin{array}{c}\text { Total de competencias } \\
\text { específicas }\end{array}$ \\
\hline Universitat de les Illes Balears & Grado en Protocolo y Organización de Eventos & 13 \\
\hline Universidad Antonio Nebrija & $\begin{array}{l}\text { Grado en Comunicación Corporativa, Protocolo y Organización } \\
\text { de Eventos }\end{array}$ & 8 \\
\hline Universidad Rey Juan Carlos & $\begin{array}{l}\text { Grado en Protocolo, Organización de Eventos y Comunicación } \\
\text { Corporativa }\end{array}$ & 7 \\
\hline Universidad Camilo José Cela & Grado en Protocolo y Organización de Eventos & 5 \\
\hline Universidad Miguel Hernández de Elche & $\begin{array}{l}\text { Grado en Organización de Eventos, Protocolo y Relaciones } \\
\text { Institucionales }\end{array}$ & 4 \\
\hline Universidad del Atlántico Medio & Grado en Protocolo y Organización de Eventos & 4 \\
\hline
\end{tabular}

Fuente: elaboración propia

En un primer análisis se observa que los grados con menor número de competencias específicas son el de la Universidad Miguel Hernández de Elche, que prioriza de entrada la organización de eventos en su denominación, y la Universidad del Atlántico Medio, cuyo plan de estudios tiene una carga menor de créditos docentes. 
Se recogen en total 41 competencias específicas. Atendiendo a la categorización en función de su objetivo, se recogen mayor número de competencias en la aplicación práctica del protocolo/ceremonial y simbología (19), seguido de los conocimientos teóricos (15) y la vinculación del protocolo y el mundo profesional/empresarial y social (7).

La Universidad con mayor enfoque en la vinculación del protocolo y el mundo profesional/empresarial y/o social sería la Universidad Nebrija, mientras que la Universidad con mayor aplicación práctica del protocolo sería la Universitat de les Illes Balears. Esta misma universidad es además la que recoge mayor número de competencias en el área teórica del protocolo.

No se registran competencias relacionadas con la investigación por lo que se sobrentiende que los trabajos fin de grado estarán directamente relacionados con la aplicación práctica del conocimiento. Hecho que también podría relacionarse con el nivel de estudios analizados.

En cuanto a las salidas profesionales cabe destacar que se recogen mayor número de menciones en el área de comunicación, seguida de eventos y después protocolo. Las profesiones más mencionadas en comunicación son Responsable/ director de comunicación y Relaciones públicas que aparecen en 4 de los 6 grados; y Director de relaciones institucionales y Gabinetes de comunicación que aparecen en el 50\% de las titulaciones. En relación al protocolo se alude a Responsable, jefe o director de protocolo en 5 de los 6 grados, seguida de consultor o asesor externo de protocolo y organización de actos/eventos y técnico y coordinador de protocolo. Las profesiones más citadas en el área de eventos son Diseño y organización de eventos / Event Manager, Director, coordinador o técnico en producción de eventos y Gestor en eventos deportivos y culturales.

\section{2. resultados: análisis de los estudios de máster}

El análisis de este trabajo recoge que en España se ofertan en estos momentos siete máster universitarios, cinco de ellos implantados, una titulación a extinguir y otra extinguida (ambas de la Universidad Católica San Antonio). Dado que la Universidad Católica San Antonio ya no oferta en su página web este título en extinción no se ha incluido en el análisis que se presenta a continuación:

Tabla 4. Resumen de las titulaciones de máster oficiales en protocolo

\begin{tabular}{|l|l|}
\hline \multicolumn{1}{|c|}{ MÁSTER } & \multicolumn{1}{|c|}{ UNIVERSIDAD } \\
\hline Máster Universitario en Comunicación Corporativa, Protocolo y Eventos & Univesidad Oberta de Catalunya \\
\hline $\begin{array}{l}\text { Máster Universitario en Dirección de Protocolo, Producción, Organización y Diseño de } \\
\text { Eventos }\end{array}$ & Universidad Camilo José Cela \\
\hline Máster Universitario en Organización de Eventos, Protocolo y Relaciones Institucionales & Universidad Miguel Hernández de Elche \\
\hline Máster Universitario en Protocolo, Comunicación y Organización Integral de Eventos & Universidad Rey Juan Carlos \\
\hline Máster Universitario en Protocolo & $\begin{array}{l}\text { Universidad Nacional de Educación a } \\
\text { Distancia (UNED) }\end{array}$ \\
\hline
\end{tabular}

Fuente: elaboración propia 
Todos los másteres presentan el término protocolo en su denominación aunque al igual que las titulaciones de grado se añade la comunicación corporativa, los eventos o las relaciones institucionales. Solo la titulación de la UNED se ciñe exclusivamente al ámbito del protocolo.

El primer máster verificado es el de la Universidad Camilo José Cela (2011), le sigue la Universidad Rey Juan Carlos (2012), la Universidad Miguel Hernández de Elche (2013), la UNED (2014) y la Universitat Oberta de Catalunya (2016). A diferencia de los estudios de grado, se recoge mayor oferta en las universidades públicas y solo una de ellas es privada. Dos de las titulaciones se adscriben a facultades de comunicación, una a la facultad de derecho y dos a centros e institutos superiores.

La Universidad Camilo José Cela es la única que ofrece las tres modalidades posibles (presencial, semipresencial y a distancia), la Universidad Miguel Hernández de Elche oferta los estudios en presencial y a distancia y la Universidad Rey Juan Carlos solo ofrece modalidad presencial. Dos de las titulaciones se imparten únicamente a distancia (UOC y UNED). Aunque se observa mayor flexibilidad en las modalidades de impartición de máster, respecto a los estudios de grado, de nuevo, Madrid es la ciudad con mayor oferta en este campo.

Los másteres ofrecen en su mayoría planes de estudio de 60 créditos, excepto la UNED que aumenta hasta los 90 ECTS, siendo por tanto la que presenta mayor porcentaje de estudios relacionados directamente con el protocolo: 83,3\%. Le siguen el máster de la Universidad Camilo José Cela (45\%) y la Universidad Rey Juan Carlos (45\%), la UOC (36,6\%), y la Universidad Miguel Hernández de Elche (30\%).

A pesar de tratarse de programas de alta especialización las asignaturas en la mayoría de las titulaciones se asemejan a las de los grados analizados aunque tienden a hibridarse y concentrarse los contenidos, lo que dificulta su categorización. Encontramos así asignaturas como "Protocolo institucional y empresarial" de 6 ECT. No obstante algunos programas como el de la UNED destacan precisamente por inclinarse hacia una alta especialización otorgando una carga de créditos similar en asignaturas diferenciadas: "Protocolo y comunicación institucional" (5 ECTS) y "Protocolo y empresa: ceremonial corporativo".

En cuanto a la categorización de las asignaturas, esta ha resultado más compleja que en el caso de grado, ya que algunos contenidos, que en origen suelen estar diferenciados, son fusionados, como se ha explicado anteriormente. En total se recogen 36 asignaturas, pero los porcentajes se han calculado sobre 37 ya que la asignatura "Protocolo institucional y empresarial" de la Universidad Rey Juan Carlos se ha incluido en dos categorizaciones:

- En el área de Historia del ceremonial y protocolo, solo uno de los programas de máster ofrece este contenido (2,7\%). Esto sugiere que los másteres tratan de ofrecer un enfoque más profesional y se alejan de los contenidos de la historia y las bases del ceremonial y protocolo.

- Igualmente, el protocolo institucional/oficial pierde peso en los programas de máster y solo aparece en tres de los másteres: dos de ellos como contenido específico y uno hibridado con el protocolo empresarial $(16,2 \%)$.

- Cuatro de las cinco universidades con máster en protocolo presentan asignaturas sobre protocolo empresarial en sus planes de estudios, representando el 10,8\% de los contenidos. 
- El Protocolo internacional y la diplomacia suponen el 16.2\% de los contenidos de los másteres de estudio, siendo el Instituto Mediterráneo de Estudios de Protocolo la institución que más protagonismo le otorga.

- El Derecho premial/nobiliario/heráldica/vexilología son los contenidos con menor peso en estas titulaciones y solo dos de los programas de máster registran asignaturas de esta área (5.4\%).

- Los contenidos con mayor representación son los relacionados con organización de eventos y protocolo que suponen el $29,7 \%$ de los contenidos de los planes de estudio analizados.

- El único máster que presenta contenidos altamente especializados en protocolo es de la Universidad Nacional de Educación a Distancia (UNED) que recoge el protocolo militar, académico, religioso y en deportes, entre otros (18.9\%).

\section{Ilustración 2 Gráfico: contenidos ofrecidos en los másteres oficiales en protocolo}

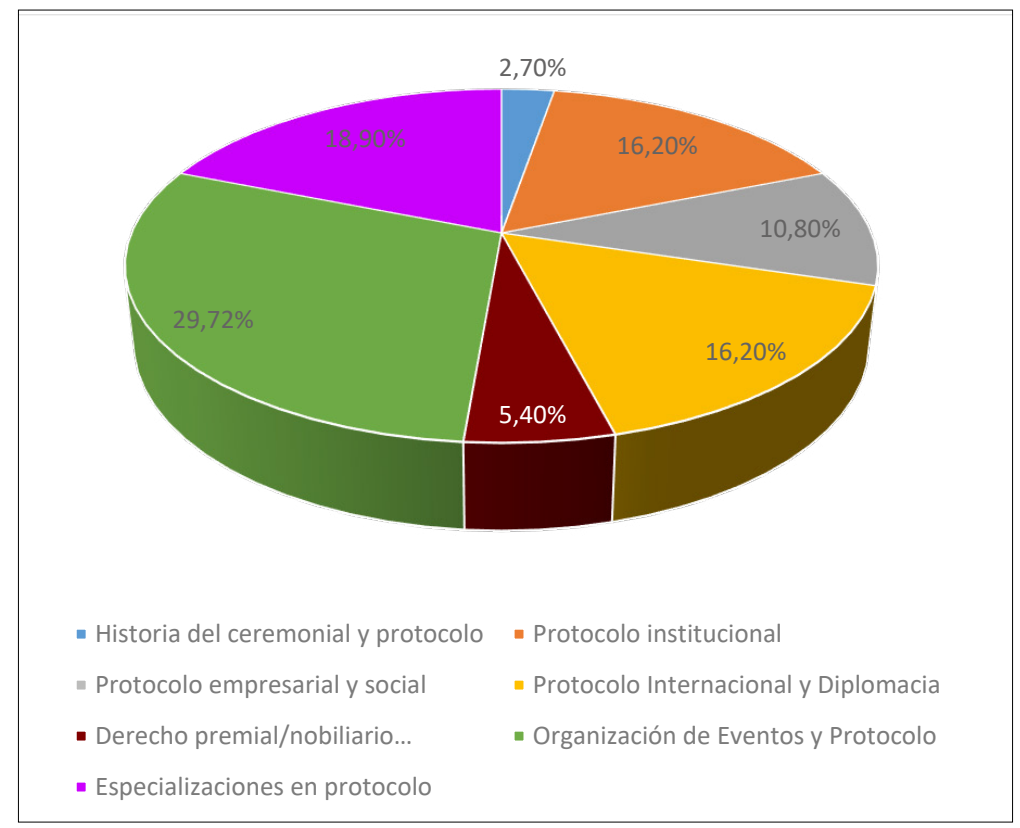

Fuente: elaboración propia 
Las competencias específicas de protocolo varían considerablemente entre las universidades:

Tabla 5. Competencias específicas en protocolo en los programas de máster

\begin{tabular}{|l|l|l|}
\hline \multicolumn{1}{|c|}{ Universidad } & \multicolumn{1}{|c|}{$\begin{array}{c}\text { Total de } \\
\text { competencias } \\
\text { específicas }\end{array}$} \\
\hline Universidad Miguel Hernández de Elche & $\begin{array}{l}\text { Máster Universitario en Organización de Eventos, } \\
\text { Protocolo y Relaciones Institucionales }\end{array}$ & 9 \\
\hline Universidad Rey Juan Carlos & $\begin{array}{l}\text { Máster Universitario en Protocolo, Comunicación y } \\
\text { Organización Integral de Eventos }\end{array}$ & 8 \\
\hline Universidad Camilo José Cela & $\begin{array}{l}\text { Máster Universitario en Dirección de Protocolo, } \\
\text { Producción, Organización y Diseño de Eventos }\end{array}$ & 6 \\
\hline Universidad Nacional de Educación a Distancia (UNED) & Máster Universitario en Protocolo & 6 \\
\hline Universidad Oberta de Catalunya & $\begin{array}{l}\text { Máster Universitario en Comunicación Corporativa, } \\
\text { Protocolo y Eventos }\end{array}$ & 1 \\
\hline
\end{tabular}

Fuente: elaboración propia

Atendiendo a esta categorización, se recogen mayor número de competencias en la aplicación práctica del protocolo/ ceremonial y simbología (15), seguido de la vinculación del protocolo y el mundo profesional/empresarial y social (7), los conocimientos teóricos (6) y la investigación (2).

El título con mayor enfoque en la vinculación del protocolo y el mundo profesional/empresarial y/o social así como con mayor aplicación práctica del protocolo sería el de la Universidad Miguel Hernández de Elche. En cuando a los conocimientos teóricos, el programa de la UNED es el que recoge mayor número de competencias.

A diferencia de los programas de grado, en máster si se recogen competencias relacionadas con la investigación, aunque solo en dos de las cinco titulaciones. En este resulta fundamental ya que los alumnos tienen que presentar en todos los casos su trabajo fin de máster que requiere necesariamente de la aplicación de la investigación en el objeto de estudio.

En máster las salidas profesionales son más dispersas que en grado, no obstante encontramos que de nuevo hay más referencias al campo de la comunicación, seguida de los eventos y luego el protocolo. La profesión más mencionada en comunicación es "Director de relaciones públicas". En el caso de protocolo encontramos "Técnico o coordinador de protocolo y relaciones institucionales" y "Jefe o técnico de protocolo"; mientras que en eventos la más repetida es "Organizador profesional de congresos (OPC)" o en su defecto "Organizador de congresos, ferias y/o reuniones".

\section{Resumen de los títulos propios}

El objeto de estudio de este trabajo se ha centrado en el análisis de titulaciones oficiales. No obstante, y con la intención de hacer una comparación cuantitativa se han recogido también una muestra de títulos propios. En este caso, y a al no tener una base de datos de consulta similar al RUCT, se utilizó para la misma la propia búsqueda de Google acotándola a las keywords "Experto en protocolo", “Máster en protocolo”, “Título propio en protocolo”, limitando las búsquedas a las 
3 primeras páginas de Google. Se ha delimitado la selección únicamente a aquellos títulos impartidos por universidades, centros adscritos y escuelas de negocio.

En total se han recogido 11 titulaciones de experto y 21 Máster en protocolo, lo que supone un total de 32 programas no oficiales. Esta cifra casi triplica el total de estudios oficiales. 22 de estos estudios, es decir el 70\%, se imparten en universidades privadas. Los estudios presenciales se imparten mayoritariamente en Madrid, seguido de Barcelona, Granada, Oviedo y Murcia.

Al igual que en los títulos oficiales, la vinculación con la organización de eventos, la comunicación y las relaciones institucionales figura en casi todos las denominaciones, aunque encontramos otras especializaciones distintas como el protocolo oficial y diplomático, la especialización en ceremonial del Estado e Internacional, en Negocios y Turismo, en eventos MICE o eventos online.

La Escuela Internacional de Protocolo (EIP) e ISEMCO son las organizaciones con mayor volumen de títulos propios, estudios que certifican en algunos casos a través de distintas universidades a pesar de tratarse de la misma empresa organizadora.

\section{Conclusiones}

A través del presente trabajo se ha analizado las características de la oferta formativa en grado y máster oficial en el área de protocolo en España. La primera conclusión es que dicha oferta sigue siendo reducida en comparación con otras campos de conocimiento, circunstancia que puede estar relacionada con la "novedad" de la oficialidad de estos estudios, ya que el primer título fue verificado en 2010 lo que supone tan solo 9 años de longevidad institucional. Si bien la expansión de las nuevas titulaciones se concentra fundamentalmente entre 2012 y 2017.

En total solo se recogen 11 titulaciones oficiales especializadas, 6 de grado y 5 de postgrado, que en comparativa con la oferta de títulos propios y títulos oficiales sigue siendo limitada. En todos ellos se vincula el protocolo con la organización de eventos y la comunicación, ofreciendo una especialización en protocolo menor en la mayoría de los casos.

La oferta de grado se concentra en universidades privadas mientras que en el caso de máster esta tendencia se invierte y se recoge mayor oferta en el ámbito público. Esta situación se enmarca en la lógica del ámbito universitario ya que las universidades privadas suelen tener mayor libertad tanto para el desarrollo de nuevas titulaciones como para la contratación de profesorado. Por su parte, la universidad pública trata de ofrecer esta especialización en postgrado, estudios que precisan de menor volumen de contratación.

De hecho, podríamos afirmar que la impulsora en España en las titulaciones oficiales en protocolo ha sido la Universidad Camilo José Cela que a partir de 2010 verifica e implanta su grado y posgrado.

Estos estudios mayoritariamente se adscriben a facultades de ciencias de la comunicación (4), institutos y centros adscritos (4) y a facultades de ciencias sociales (3). 
En el caso de los estudios de posgrado, se observa además que tres de las universidades parecen dar continuidad a sus grados a través de programas con el mismo nombre o similar. No obstante, estas especializaciones son muy finitas y se recurre de nuevo a contenidos, como se avanzado anteriormente, de eventos y protocolo.

Tanto los programas de grado como los de máster centran sus contenidos en los eventos, presentan más competencias en la aplicación práctica del protocolo y muestran más salidas profesionales en comunicación. Prácticamente no hay diferencias entre ambos tipos de estudio, aunque se presume que máster debería ser más especializado. Si bien, estos últimos son más flexibles en la modalidad de impartición, en cuanto a la presencialidad, Madrid es la ciudad con más oferta formativa

En relación al desarrollo idiomático, todos los estudios viran hacia la formación exclusivamente en español. Tan solo una universidad ofrece la posibilidad de cursar los estudios de grado en inglés. Circunstancia que otras universidades tratan de resolver incorporando asignaturas específicas de inglés en el área. Destaca el caso de una universidad que oferta también catalán para eventos.

Las nuevas tecnologías e internet están prácticamente ausentes en los planes de estudio o al menos, no se vinculan directamente al protocolo. No hay ninguna asignatura cuyo nombre recoja la "Netiqueta" o los eventos en el ámbito digital. El análisis de las competencias refleja que los estudios tienden a enfocarse en la aplicación práctica del protocolo por delante del enfoque teórico, de su vinculación al ámbito profesional o de la investigación. En este último punto cabe destacar que ninguno de los grados que se ofertan en la actualidad presenta competencias relacionadas. Si bien los estudios en protocolo son muy recientes, se hace necesario generar nuevas investigaciones y literatura científica que sigan asentando tanto la profesión como su conocimiento y desarrollo.

Resulta especialmente llamativo el volumen de títulos propios, experto y posgrado que se ofertan en el área. Esta circunstancia puede estar relacionada con la facilidad de su implantación ya que no precisa de verificación, seguimiento o acreditación por parte de ANECA. Así mismo, no es necesario que el profesorado sea doctor ni se exige un número mínimo de doctores acreditados. Este amplio volumen de oferta opaca a los títulos propios que deberían de aprovechar este valor añadido en su posicionamiento.

Igualmente y de cara a un futuro, cabe la reflexión por parte de las universidades públicas en cuanto a la implantación de estos títulos, que suponen un grado de especialización de valor.

Finalmente y con esta radiografía, el protocolo parece posicionarse en los estudios oficiales como una herramienta al servicio de la organización de eventos y la comunicación. Sigue siendo necesario revalorizar estos estudios, incluyendo en los itinerarios de máster contenido más especializado, ampliando las competencias y asignaturas de investigación en todos los estudios; y tratado a través de ambas acciones de incentivar la investigación en el campo para que un futuro este mismo ejercicio nos muestre que los estudios oficiales han crecido junto con la demanda de profesionales especializados. 


\section{Referencias bibliográficas}

Agencia Nacional de Evaluación de la Calidad y Acreditación (2003). Libro Blanco. Títulos de Grado en Comunicación. Aneca.es. En: http://www.aneca.es/var/media/150336/libroblanco_comunicacion_def.pdf (Consultado el 26 de marzo de 2019).

Álvarez, M. L. (2008). Nociones de protocolo desde la bibliografía de sus autoridades, en Revista Latina de Comunicación Social, 63, consultado el 22 de diciembre de 2017, disponible en http://www.ull.es/publicaciones/latina/_2008/15_08_ Vigo/ML_Alvarez_Rodriguez.html

Asociación de Directivos de Comunicación (2018). Estado de la comunicación en España en Anuario de la comunicación 2018. Madrid: DIRCOM.

Asociación de la prensa de Madrid (2017). Informe anual de la profesión periodística. Apmadrid.es. En: https://www. apmadrid.es/publicaciones/informe-anual-de-la-profesion/ Consultado el 27 de marzo de 2019.

Barquero; J. D.; Fernández, F. (2007). Los secretos del protocolo, las relaciones públicas y la publicidad. Valladolid: Lex Nova.

Borau, E. (2017). El protocolo y su importancia en la gestión de la comunicación corporativa en Perlado, M.; Cachán, C. (2017) Competencias y perfiles profesionales en el ámbito de la comunicación. Madrid: Dykinson.

Castillo-Esparcia, A. y Fernández-Torres, M. J. (2015). Protocolo y relaciones públicas, Madrid: Editorial Síntesis.

Castillo, A. (2009). Relaciones públicas: teoría e historia. Barcelona: UOC.

Evento Plus (2018). La recuperación del sector de reuniones y eventos se consolida. Eventoplus.com. En: https://www. eventoplus.com/noticias/la-recuperacion-del-sector-de-reuniones-y-eventos-se-consolida/ Consultado el 27 de marzo de 2019.

Fernández, F; Barquero, J. D. (2004). El Libro azul del protocolo y las relaciones públicas. Madrid: McGraw-Hill.

González, H. (2017). El protocolo, una herramienta de comunicación que no deja nada al azar. ElDiario.es. Consultado el 04 de enero de 2017. Disponible en: http://www.eldiario.es/cultura/protocolo-herramienta-comunicacion-deja-azar_0_648485400.html

Herrero, J.C.; Perelló, M. M. (2016). La enseñanza del protocolo y la organización de eventos en el espacio europeo de educación superior: análisis comparado de los planes de estudio. Estudios sobre el Mensaje Periodístico, 23(1), 437-455. doi: http://dx.doi.org/10.5209/ESMP.55606

Corbacho, J. M. (2013). El Protocolo en la docencia universitaria: la materia de Relaciones Públicas y Protocolo en el marco de la adaptación al Espacio Europeo de Educación Superior. Estudios sobre el Mensaje Periodístico, 19, pp. 121-128.

Hernández, S.; Losada, J. C.; Matilla, K. (2009). Las relaciones públicas y la comunicación corporativa en la oferta universitaria de postgrado del Estado Español: análisis sistemático y proyectivo y una iniciativa de observación del Estado de la situación por parte de una asociación profesional. Razón y palabra, 70. ISSN-e 1605-4806. 
Herrero, L. (2013). La industria creativa de la Organización de Eventos y el Protocolo. Análisis bibliométrico de contribuciones académicas. TFM Universidad de Alicante. Disponible en: http://rua.ua.es/dspace/handle/10045/34020 Consultado el 22 de marzo de 2019

International Congress and Convention Association (2017). The International Association Meetings Market 2017. iccaworld.org. En: https://www.iccaworld.org/knowledge/benefit.cfm?benefitid=4036 Consultado el 27 de marzo de 2019. López-Nieto, Mallo, F. (2006). Honores y protocolo. Madrid: El Consultor de Los Ayuntamientos.

Marín Calahorro, F. (2000). Protocolo y Comunicación. Los Medios en los Actos Públicos. Barcelona: Bayer Hnos. S.A

Moreno, D. (2019). Andrés Virto, nuevo presidente de Agencias de Eventos Españolas Asociadas (AEVEA): "No se puede negar que existe competencia desleal en España”. El Publicista, 399, pp.28-30.

Otero, M. T. (2002). Las funciones del ceremonial y protocolo en la reputación corporativa. Sphera Pública, 2, 135-147.

Otero, M. T. (2009). Protocolo y organización de eventos. Barcelona: Editorial UOC.

Perlado, M.; Saavedra, M. (2017). Los estudios universitarios especializados en comunicación en España. Barcelona: UOC.

Portugal; M. C.; Becerra, L.; Victoria, J. S.; (2016). El protocolo y el ceremonial como disciplina académica: análisis de los estudios universitarios oficiales en España. Estudios Institucionales, 3(5), 137-156.

Sánchez, D. (2017). Fundamentos del ceremonial y del protocolo. Madrid: Síntesis.

Sánchez, D. (2017). Manual de protocolo oficial y derecho ceremonial del Estado. Madrid: Síntesis.

Sierra, J.; Sotelo, J. (2008). El estado actual del protocolo a nivel jurídico y profesional. Revista de Comunicación y Nuevas Tecnologías Icono14, 11, pp. 1g-34.

Vilarrubias, F. (2005). Derecho premial: Protocolo, Ceremonial, Heráldica y Vexilología en el Estado. Oviedo: Universidad de Oviedo. 